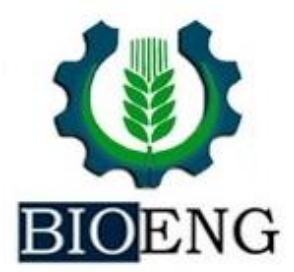

\title{
AVALIAÇÃO DOS SUBPRODUTOS DE DEGRADAÇÃO DO HERBICIDA AMETRINA OBTIDOS VIA PROCESSOS OXIDATIVOS AVANÇADOS
}

${ }^{1}$ USP - Universidade de São Paulo, Departamento de Ciências Básicas e Ambientais, Escola de Engenharia de Lorena, Lorena, SP, Brasil

${ }^{2}$ USP - Universidade de São Paulo, Departamento de Química e Física Molecular, Instituto de Química de São Carlos, São Carlos, SP, Brasil

${ }^{3}$ UNICAMP - Universidade Estadual de Campinas, Departamento de Engenharia de Materiais, Faculdade de Engenharia Mecânica, Campinas, SP, Brasil

${ }^{4}$ USF - Universidade São Francisco, Grupo de Pesquisas em Meio Ambiente e Sustentabilidade, Itatiba, SP, Brasil

Article history: Received 17 January 2018; Received in revised form 10 March 2018; Accepted 12 March 2018; Available online 27 March 2018.

\section{RESUMO}

As tecnologias alternativas, como os processos oxidativos avançados (POA), contribuem para o controle das poluições ambientais e nesse contexto uma das informações mais importantes é a determinação dos subprodutos de degradação formados em cada condição de estudo. Nesse trabalho foram avaliadas diferentes concentrações de $\mathrm{H}_{2} \mathrm{O}_{2}, \mathrm{pH}$ e temperatura, sempre com o objetivo de promover a quebra da molécula do herbicida triazínico ametrina e avaliar os principais subprodutos formados no processo de degradação. O maior número de subprodutos foi identificado utilizando a espectrometria de massas com inserção direta nas condições com $20 \%$ e $25 \%$ de $\mathrm{H}_{2} \mathrm{O}_{2}$ a $65^{\circ} \mathrm{C}$, sendo detectados 5 possíveis compostos originados a partir da fragmentação da molécula original da ametrina.

Palavras-chave: Herbicida, Ametrina, processo oxidativo avançado, degradação química

\section{EVALUATION OF DEGRADATION BY-PRODUCTS OF HERBICIDE AMETRIN OBTAINED BY ADVANCED OXIDATIVE PROCESSES}

\begin{abstract}
Alternative technologies, such as advanced oxidative processes (AOP), contribute to the control of environmental pollution and in this context one of the most important information is the determination of degradation by-products formed in each study condition. In this work, different $\mathrm{H}_{2} \mathrm{O}_{2}$ concentrations, $\mathrm{pH}$ and temperature were evaluated, with the objective of promoting the breakdown of the molecule of the triazine herbicide ametryn and to evaluate the main by-products formed in the degradation process. The largest number of by-products was identified using direct insertion mass spectrometry under conditions with $20 \%$ and $25 \%$ $\mathrm{H}_{2} \mathrm{O}_{2}$ at $65^{\circ} \mathrm{C}$. Five possible compounds were detected from the fragmentation of the original ametryne molecule.
\end{abstract}

Keywords: Ametrine, degradation by-products, advanced oxidative process, chemical degradation

robson.rocha@usp.br 


\section{INTRODUÇÃO}

$\mathrm{O}$ crescimento de centros urbanos e de áreas industrializadas é um dos grandes fatores responsáveis pela contaminação do meio ambiente, assim como o acúmulo e a dispersão de poluentes gerados por esse crescimento podem causar efeitos adversos à saúde humana e de outras espécies (FARRÉ 2002).

Segundo a Abrasco (Associação Brasileira de Saúde Coletiva), em 2016, $70 \%$ dos alimentos in natura consumidos no país estavam contaminados por resíduos de agrotóxicos e segundo a Anvisa (Agência de Vigilância Sanitária) 28\% continham substâncias não autorizadas (INPE 2016).

Devido ao aumento da população mundial houve um aumento significativo na demanda por alimentos e, consequentemente, uma maior demanda por uma produção agrícola mais eficiente, com menor incidência de doenças e pragas nas áreas de cultivo (FARRÉ 2002). Mas para alcançar essa eficiência, os produtores recorrem ao uso de agrotóxicos, compostos com elevada toxicidade para o homem e para o meio ambiente (FARRÉ 2002).

De acordo com levantamento realizado em 2015 pela Agência EMBRAPA de Informação Tecnológica, anualmente são consumidos aproximadamente 2,5 milhões de toneladas de agrotóxicos no mundo, sendo que no Brasil, o consumo é superior a $300 \mathrm{mil}$ toneladas de agrotóxico (SPADOTO 2015).

No Brasil a culturas que mais se utiliza agrotóxicos são: soja, milho, citros, cana-de-açúcar. As regiões brasileiras que mais consomem estes produtos químicos são sudeste $(38 \%)$, sul $(31 \%)$ e centrooeste (23\%), sendo que São Paulo consome aproximadamente $25 \%$ dos agrotóxicos utilizado no Brasil (SPADOTO 2015).

Considerando os agrotóxicos mais utilizados, pode-se citar os herbicidas, que são amplamente consumidos devido às suas características, apresentando um amplo espectro de ação e eficiência em diversas culturas. Dentre as culturas que mais utilizam esta classe de agrotóxicos está a de cana-de-açúcar, sendo um dos compostos mais utilizados é o 2-etilamina4-isopropilamina-6-metiltio-s-triazina, mais conhecido como ametrina (AME). A AME é um agrotóxico pertencente ao grupo dos triazínicos. Segundo Farré (2002) a AME é um herbicida que apresenta elevada solubilidade em água, elevada toxicidade para crustáceos e moluscos, porém com baixa toxicidade para mamíferos e peixes, o que não significa que não apresente dano a saúde do ser humano (FARRÉ 2002). Embora alguns agrotóxicos sejam amplamente estudados, muitos ainda não possuem informações suficientes quanto aos mecanismos de toxicidade e possíveis efeitos em diferentes doses e vias de exposição e principalmente quanto aos seus metabólitos ou subprodutos de degradação.

A AME pode apresentar ainda, movimentação lateral e vertical no solo, podendo ser lixiviada por chuvas, corpos d'agua subterrâneos ou mesmo pela irrigação, podendo alcançar grandes extensões de solo (FARRÉ 2002). Além da contaminação do meio ambiente pela AME ser agravada pela sua movimentação no solo, observa-se também, um agravamento pelo método de aplicação deste herbicida, onde apenas uma parte atinge efetivamente o alvo específico, a planta; enquanto outra parte atinge o solo diretamente e assim, juntamente com o resíduo lixiviado pode atingir corpos d'água (FAVA 2007; JABLONOWSKI 2008; DICTOR 2008; ELIMACHE 2009; NOGUEIRA 2002; NOGUEIRA 2004; NOGUEIRA 2007; TROVÓ 2005).

Este processo de transferência do poluente para o meio ambiente é agravado pela quantidade de herbicida comercializado. Segundo Rezende (2011), o Brasil apresenta um elevado consumo de herbicidas, principalmente nas áreas do 
centro-sul do país, nas culturas de soja, milho e cana-de-açúcar (REZENDE 2001). Associando à quantidade de herbicida consumida com a parcela transferida diretamente para o solo, pode-se verificar a grande quantidade desses compostos presentes no meio ambiente, seja no solo ou no meio aquático (EL IMACHE 2009; JABLONOWSKI 2008). No cultivo da cana-de-açúcar o uso de herbicidas ultrapassa os $1 \mathrm{~kg} \mathrm{ha}^{-1}$, o que intensifica contaminação de águas subterrâneas e/ou de superfície e aumenta a necessidade de tratamento dessas águas, pois contaminantes como a AME possuem elevado tempo de meia-vida no meio ambiente (FARRÉ 2002).

Considerando as potencialidades de contaminação pelos herbicidas, a água é o ambiente que mais apresenta contaminação por estes compostos. Através da água, estes compostos podem chegar a um grande número de seres vivos, direta ou indiretamente e dependendo da concentração ou bioacumulação ao longo da cadeia alimentar. Os herbicidas podem causar sérios danos à saúde, sendo assim é necessário o estudo e desenvolvimento de novas tecnologias eficazes no tratamento de águas contaminadas por agrotóxicos visando à prevenção dos diversos ecossistemas (REZENDE 2001; NOGUEIRA 2002; NOGUEIRA 2004).

Com a potencialidade de contaminação do meio ambiente por herbicidas, a literatura apresenta diversos trabalhos sobre técnicas de tratamento de águas contaminadas com tais compostos, incluindo a AME, as quais envolvem o uso de processos de biodegradação (FARRÉ 2002), uso de bio-reatores (NAVARATNA 2012) e sistemas de tratamento utilizando técnicas foto-assistidas (REBELO 2007).

Considerando as técnicas de tratamento de águas contaminadas com compostos orgânicos descritas na literatura, o uso de reagentes oxidantes se destaca, por exemplo, espécies de cloro ativo e peróxido de hidrogênio são utilizados em vários sistemas de tratamento (GAO 2009; XU 2009).

A utilização do poder oxidante do radical hidroxila $\left({ }^{\circ} \mathrm{OH}\right)$ no processo de tratamento, obtido a partir do peróxido de hidrogênio $\left(\mathrm{H}_{2} \mathrm{O}_{2}\right)$, ocorre nos chamados Processos Oxidativos Avançados (POA). Segundo Nogueira (2009), estes processos são alternativos aos processos clássicos de tratamento de efluentes, uma vez que gera radicais hidroxila, altamente reativos e pouco seletivos, podendo atuar na oxidação química de uma vasta gama de substâncias orgânicas e recalcitrantes, inclusive os herbicidas (NOGUEIRA 2002; NOGUEIRA 2004).

Nos POA, o reagente Fenton têm se destacado pela simplicidade de aplicação, rapidez e eficiência na degradação de diversos compostos orgânicos, sendo caracterizado essencialmente pela geração do radical hidroxila em meio ácido na faixa de $\mathrm{pH}$ ideal 2,8-3,0 (NOGUEIRA 2007; TROVÓ 2005).

\section{Processo de degradação utilizando Reagente Fenton}

$\mathrm{O}$ uso de $\mathrm{H}_{2} \mathrm{O}_{2}$ com íons $\mathrm{Fe}^{2+}$ em meio ácido (Processo Fenton) é uma das técnicas mais estudadas na literatura para degradação de compostos orgânicos em meio aquoso (NOGUEIRA 2002; NOGUEIRA 2004; NOGUEIRA 2007; TROVÓ 2005; MASCOLO 1994; XU 2009; GRILLO 2012). Segundo Oturan (2011), o ${ }^{\circ} \mathrm{OH}$ é utilizado na oxidação de compostos orgânicos, podendo levar a completa mineralização do composto orgânico em determinadas condições e pode ser obtido a partir de diferentes técnicas, como o Processo Fenton, onde essa espécie radicalar é gerada a partir do $\mathrm{H}_{2} \mathrm{O}_{2}$ na presença de íons $\mathrm{Fe}^{2+}$, de acordo com a equação 1 (OTURAN 2011; NEYENS 2003; WALLING 1975; WALLING 1973):

$\mathrm{Fe}^{2+}+\mathrm{H}_{2} \mathrm{O}_{2} \rightarrow \mathrm{Fe}^{3+}+{ }^{\bullet} \mathrm{OH}+{ }^{-} \mathrm{OH}$ 
Os íons $\mathrm{Fe}^{2+}$ iniciam a decomposição do $\mathrm{H}_{2} \mathrm{O}_{2}$, resultando na geração do ${ }^{\circ} \mathrm{OH}$ que é um oxidante mais forte que $\mathrm{o} \quad \mathrm{H}_{2} \mathrm{O}_{2} \quad$ (OTURAN 2011. Segundo Walling, a formação de ${ }^{\bullet} \mathrm{OH}$ em meio aquoso envolve uma complexa sequência de reações, onde o excesso de $\mathrm{H}_{2} \mathrm{O}_{2}$ pode promover as equações 2 e 3 WALLING 1975; WALLING 1973):

$$
\begin{aligned}
& \mathrm{Fe}^{3+}+\mathrm{H}_{2} \mathrm{O}_{2} \rightarrow \mathrm{Fe}-\mathrm{OOH}^{2+}+\mathrm{H}^{+} \\
& \mathrm{Fe}-\mathrm{OOH}^{2+} \rightarrow{ }^{\cdot} \mathrm{O}_{2} \mathrm{H}+\mathrm{Fe}^{2+}
\end{aligned}
$$

O processo de catálise da formação do ${ }^{\circ} \mathrm{OH}$ em excesso de $\mathrm{H}_{2} \mathrm{O}_{2}$ é lento e forma um radical $\left({ }^{\circ} \mathrm{O}_{2} \mathrm{H}\right)$ menos oxidante quando comparado ao ${ }^{\circ} \mathrm{OH}$, fato que pode diminuir a eficiência da degradação de compostos orgânicos (OTURAN 2011). Nesse sentido, pode ocorre à diminuição da eficiência do processo devido a outras reações podem ocorrer paralelamente, conforme as equações 4 (consumo de $\mathrm{H}_{2} \mathrm{O}_{2}$ ) e 5 (consumo de $\mathrm{Fe}^{2+}$ ), entre outras NEYENS 2003; WALLING 1975; WALLING 1973; ZALOUK 2009):

$$
\begin{aligned}
& \bullet \mathrm{OH}+\mathrm{H}_{2} \mathrm{O}_{2} \rightarrow \mathrm{H}_{2} \mathrm{O}+{ }^{\bullet} \mathrm{O}_{2} \mathrm{H} \\
& { }^{\bullet} \mathrm{OH}+\mathrm{Fe}^{2+} \rightarrow \mathrm{Fe}^{3+}+{ }^{-} \mathrm{OH} \\
& \mathrm{R}^{\bullet}+\mathrm{O}_{2} \rightarrow \mathrm{ROO}^{\bullet} \\
& \mathrm{ROO}^{\bullet}+{ }^{\bullet} \mathrm{OH} / \mathrm{O}_{2} \rightarrow \mathrm{xCO}_{2}+\mathrm{yH}_{2} \mathrm{O} \\
& \mathrm{Ar}{ }^{\bullet}+{ }^{\bullet} \mathrm{OH} \rightarrow \mathrm{ArHO} \\
& \mathrm{ArHO}^{\bullet}+{ }^{\bullet} \mathrm{OH} / \mathrm{O}_{2} \rightarrow \mathrm{xCO}_{2}+\mathrm{yH}_{2} \mathrm{O}
\end{aligned}
$$

Segundo Brillas (2009) e Oturan (2000), o radical ${ }^{\circ} \mathrm{OH}$ pode oxidar a matéria orgânica por diferentes mecanismos, como a deshidrogenação, reações redox ou hidroxilação. Essas reações geram espécies radicalares, que podem ocorrer com a adição de oxigênio molecular (equação 6) ou com o próprio ${ }^{\circ} \mathrm{OH}$ (equação 8 ). Com a formação de espécies, na sequencia ocorre a as reações oxidativas, que dependendo das condições experimentais levará à completa mineralização, isto é, geração de $\mathrm{CO}_{2}$ e $\mathrm{H}_{2} \mathrm{O}$ como produto final, como pode ser observado nas equações 7 e 9 (BRILLAS 2009; OTURAN 2000).

\section{Avaliação dos produtos de degradação}

Diversos processos de degradação de compostos orgânicos baseados em diferentes técnicas de tratamento são descritos na literatura (NOGUERIA 2002; NOGUEIRA 2004; NAVARATNA 2012; MASCOLO 1994; XU 2009), porém, é necessário analisar os compostos formados pelos processos de degradação, pois podem gerar produtos mais tóxicos que composto original.

Avaliando o processo de degradação de herbicidas, Mascolo (1994) estudou reações de degradação com o ácido hipocloroso e dióxido de cloro. Segundo os autores, as condições experimentais indicaram que todas as triazinas investigadas reagem da mesma forma com cada espécie oxidante (MASCOLO 1994). Em 2009, Xu e colaboradores verificaram que a cinética de degradação da AME em meio de ácido hipocloroso é determinada como sendo de segunda ordem, além de estabelecer um mecanismo de degradação com a geração de subprodutos oxigenados, ligados ao átomo de enxofre, onde posteriormente este agrupamento é substituído pelo grupamento hidroxila (XU 2009). Segundo Farré (2002) a AME apresenta dois principais subprodutos de $\mathrm{m} / \mathrm{z} 198$ e 97, os quais foram identificados como sendo, respectivamente, $N$-etil- $N^{\prime}-(1-$ metil-etil)-6-(hidróxi)-1,3,5-triazina-2,4diamina e 1,3,5-triazina-2-amina, que são classificados como metabólitos por serem obtidos via processo biodegradação (FARRÉ 2002).

Segundo a Legislação Brasileira (CONAMA n 357 2005), o tratamento de águas ou efluentes contaminados com agrotóxicos deve reduzir a concentração destas substâncias à valores estipulados, porém um parâmetro importante ainda não é considerado na avaliação das técnicas de 
tratamento, os produtos formados ao longo do processo de degradação. Tais processos de degradação podem gerar compostos com diferentes toxicidades e atividade biológica para o meio ambiente, assim, é fundamental a determinação dessas estruturas em cada tratamento e considerar essa informação na avaliação da aplicabilidade da técnica.

Diante do cenário de extensa contaminação por agrotóxicos e a

\section{MATERIAIS E MÉTODOS}

O estudo de determinação e identificação de subprodutos formados foi realizado por Espectrometria de Massas de durante o processo de degradação do herbicida AME, de acordo com a adição de diferentes quantidades de $\mathrm{H}_{2} \mathrm{O}_{2}$ em diferentes valores de temperatura e $\mathrm{pH}$. Para os ensaios de degradação preparou-se uma solução padrão de AME (SigmaAldrich) com concentração de $100 \mathrm{mg} \mathrm{L}^{-1}$, que foi adequadamente separada em tubos de ensaio hermeticamente fechados, cada um contendo o volume final de $10 \mathrm{~mL}$.

Os ensaios de degradação da AME foram realizados em dois grupos de experimentos: sem e com adição de $\mathrm{H}_{2} \mathrm{O}_{2}$. $\mathrm{O}$ primeiro grupo de experimentos, sem adição de $\mathrm{H}_{2} \mathrm{O}_{2}$, foram realizados nas temperaturas de $25^{\circ} \mathrm{C}$ e $65^{\circ} \mathrm{C}$ e nos $\mathrm{pH} 1,3$

\section{RESULTADOS E DISCUSSÃO}

Considerando o processo oxidativo avançado, a degradação da molécula da AME pode ocorrer por vários mecanismos diferentes, formando diferentes produtos dependendo das condições experimentais. Dessa forma foram avaliados os produtos de degradação na ausência e na presença de $\mathrm{H}_{2} \mathrm{O}_{2}$ e a influência da temperatura e do $\mathrm{pH}$.

\section{Degradação da AME na ausência de $\mathrm{H}_{2} \mathrm{O}_{2}$}

O primeiro grupo de experimentos consistiu em avaliar a degradação da AME em diferentes valores de temperatura e $\mathrm{pH}$ diversidade de técnicas de tratamento de águas contaminadas com estes compostos, este artigo tem por objetivo avaliar a oxidação química da $\mathrm{AME}$, utilizando o Reagente de Fenton em diferentes condições experimentais de $\mathrm{pH}$ e temperatura, com o intuito de identificar os subprodutos de degradação, utilizando-se a técnica de espectrometria de massas com ionização por electrospray (ESI) com inserção direta de amostra.

(acidificado com $\mathrm{H}_{2} \mathrm{SO}_{4}$ ) e 7 . O segundo grupo de experimentos foram realizados com diferentes quantidades de $\mathrm{H}_{2} \mathrm{O}_{2}$, nas mesmas condições de temperatura e $\mathrm{pH}$ e com adição de $10 \mathrm{mmol} \mathrm{L}^{-1}$ de $\mathrm{FeSO}_{4}$.

Em ambos os grupos de experimentos, os tubos de ensaio permaneceram 10 horas sob constante agitação, com amostragens a cada 15 minutos, durante a primeira hora, e a cada 30 minutos a partir da segunda hora e ao final de $10 \mathrm{~h}$ de experimento. As amostras foram adequadamente extraídas e em seguida analisadas em um Espectrômetro de Massas com módulo de ionização por Eletrospray (ISE) da Apllied Biosystems modelo Q-trap Triplo Quadrupolar híbrido com trap linear em Q3.

com o objetivo de simular a degradação desse composto em diferentes tipos de solo e assim determinar quais os possíveis produtos podem ser formandos no solo em função destes meios, excluindo possíveis processos de biodegradação.

A análise dos espectros obtidos durante os experimentos de degradação da AME sob variação de temperatura $\left(25^{\circ} \mathrm{C}\right.$ e $\left.65{ }^{\circ} \mathrm{C}\right)$ e $\mathrm{pH}(1,3$ e 7$)$, não apresentaram diminuição da intensidade da AME $(\mathrm{m} / \mathrm{z}$ $228,7)$ e também não ocorreu a detecção de outros fragmentos, independente da temperatura ou do $\mathrm{pH}$ ao longo de $10 \mathrm{~h}$ de 
experimento. Esse resultado condiz com uma das principais características desse tipo de composto orgânico: a sua estabilidade química. Uma vez aplicadas nas culturas, as moléculas da AME devem manter sua estrutura química original e consequentemente sua atividade independente das condições do meio (GRILLO 2012).

De acordo com os experimentos realizados em diferentes valores de temperatura e $\mathrm{pH}$ não ocorreu a degradação da AME, possivelmente devido a estabilidade da sua estrutura química, assim, foram realizados experimentos de degradação na presença de $\mathrm{H}_{2} \mathrm{O}_{2}$ e íons $\mathrm{Fe}^{2+}$.

\section{Degradação da AME na presença de $\mathrm{H}_{2} \mathrm{O}_{2}$}

O segundo grupo de experimentos consistiu em avaliar a degradação da AME em diferentes valores de temperatura, $\mathrm{pH}$ na presença de $\mathrm{H}_{2} \mathrm{O}_{2}$ e íons $\mathrm{Fe}^{2+}$. Estes experimentos tiveram como objetivo simular o tratamento de águas contaminadas com AME, originadas de limpeza e manutenção de diferentes equipamentos utilizados na aplicação do pesticida também e da água originada da limpeza dos recipientes de armazenamento, assim como determina a legislação brasileira (CONAMA 2005).

De acordo o processo de formação de ${ }^{\bullet} \mathrm{OH}$ a partir dos reagentes de Fenton e sua ação na oxidação de compostos orgânicos, observa-se a importância de estabelecer a quantidade dos reagentes de Fenton $\left(\mathrm{H}_{2} \mathrm{O}_{2}\right.$ e íons $\left.\mathrm{Fe}^{2+}\right)$ para uma eficiente degradação. Assim foram utilizadas diferentes quantidades de $\mathrm{H}_{2} \mathrm{O}_{2}$ seguindo as proporções estequiométricas descritas nas Equações 10 a 12, na presença de $10 \mathrm{mmol} \mathrm{L}^{-1}$ de $\mathrm{FeSO}_{4}$, para degradação da AME. Entretanto, este processo não tem como objetivo a mineralização total dos compostos orgânicos presentes nas amostras, mas sem identificar as estruturas dos intermediários formados a partir do ataque do ${ }^{\circ} \mathrm{OH}$ na molécula da AME.

Para definição das quantidades de $\mathrm{H}_{2} \mathrm{O}_{2}$ a serem utilizadas no processo de oxidação da AME $\left(\mathrm{C}_{9} \mathrm{H}_{17} \mathrm{SN}_{5}\right)$ foi considerada a quantidade de $\mathrm{O}_{2}$ necessária para sua mineralização total. Segundo a equação 10 , são necessários $22,75 \mathrm{mols}$ de $\mathrm{O}_{2}$ para cada 1 mol de $\mathrm{AME} e$ consequentemente 45,5 mols de $\mathrm{H}_{2} \mathrm{O}_{2}$ (equação 11) para esse processo. Assim, para a reação estequiométrica de combustão da AME (equação 12) são necessários 45,5 mols de $\mathrm{H}_{2} \mathrm{O}_{2}$, sendo esta a referencia de quantidade para o processo de oxidação da AME.

$$
\begin{aligned}
& \mathrm{C}_{9} \mathrm{H}_{17} \mathrm{SN}_{5}+22,75 \mathrm{O}_{2} \rightarrow 9 \mathrm{CO}_{2}+8,5 \\
& \mathrm{H}_{2} \mathrm{O}+\mathrm{SO}_{4}{ }^{2-}+5 \mathrm{NO}_{3}{ }^{-} \\
& 45,5 \mathrm{H}_{2} \mathrm{O}+22,75 \mathrm{O}_{2} \rightarrow 45,5 \mathrm{H}_{2} \mathrm{O}_{2} \\
& \left.\mathrm{C}_{9} \mathrm{H}_{17} \mathrm{SN}_{5}+411\right) \\
& \mathrm{H}_{2} \mathrm{O}+\mathrm{SO}_{4}{ }^{2-}+5 \mathrm{H}_{2} \mathrm{O}_{2}-9 \mathrm{NO}_{3}{ }^{-}
\end{aligned}
$$

Diante da relação entre 45,5 mols de $\mathrm{H}_{2} \mathrm{O}_{2}$ para cada mol de AME, foram realizados experimentos variando de $10 \%$ a $100 \%(\mathrm{~m} / \mathrm{m})$ da quantidade da espécie oxidante, descrita na equação 12 , para avaliar a formação de produtos da oxidação da AME.

Para a análise dos subprodutos de oxidação da AME, deve-se primeiramente conhecer quais os produtos formados pela fragmentação da molécula da AME no espectrômetro de massas. Inicialmente foi analisada uma amostra padrão da AME para determinação de seus principais íons moleculares formados de acordo com a fragmentação da AME, os quais são apresentados na Figura 1: 


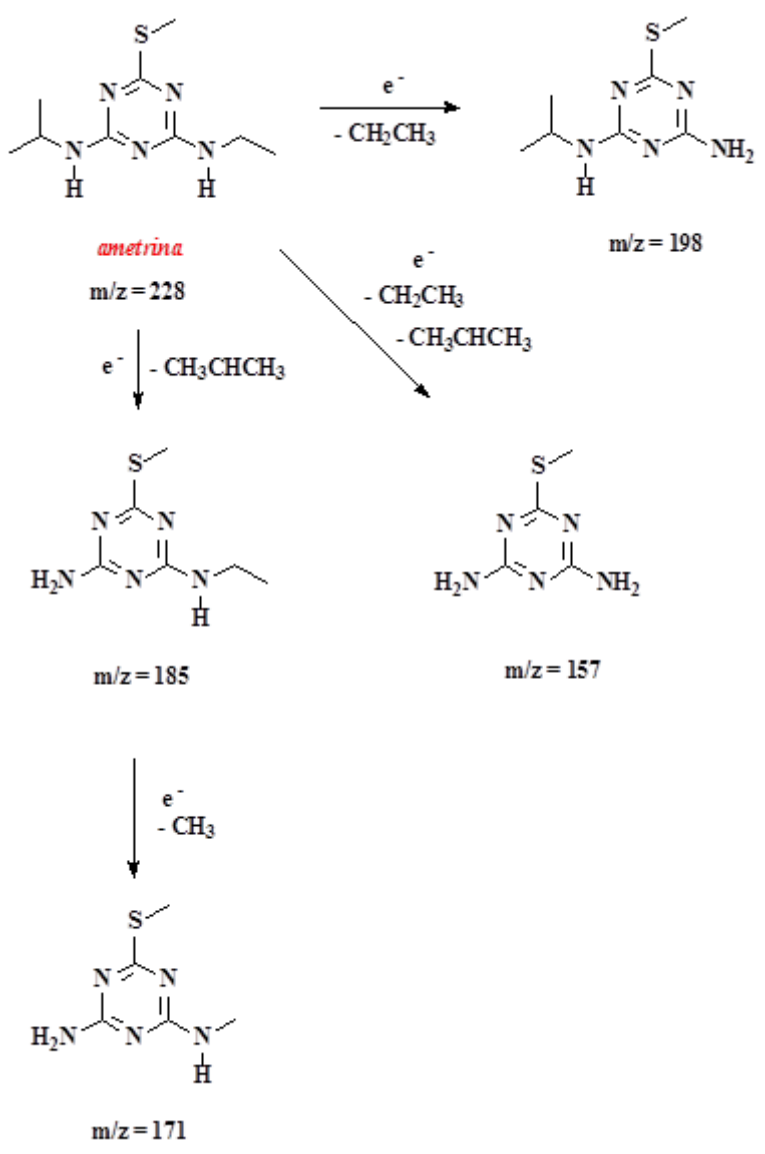

Figura 1. Fragmentação do padrão da ametrina analisada no EM-ESI.

A mais importante característica de fragmentação da molécula da AME quando submetida a um feixe de elétrons podem ser formadas diferentes estruturas devido à perda de grupamentos como: $\mathrm{CH}_{3}, \mathrm{C}_{2} \mathrm{H}_{5}$, $\mathrm{C}_{3} \mathrm{H}_{7}$. A Figura 1 descreve as sucessivas fragmentações que podem ocorrem na molécula da AME, resultando na saída de grupos descritos acima. Esta etapa de identificação é importante, para determinar as estruturas correspondentes à fragmentação da AME.

Para a análise dos produtos formandos na oxidação da AME foram realizados experimentos nos $\mathrm{pH} 1,3$ e 7 , na temperatura de $65^{\circ} \mathrm{C}$ e com $20 \%$ e $25 \%$ de $\mathrm{H}_{2} \mathrm{O}_{2}$, sendo que as demais condições de oxidação propostas apresentaram condições desfavoráveis a identificação dos subprodutos formandos. Nos experimentos a $25^{\circ} \mathrm{C}$ e com quantidades de $\mathrm{H}_{2} \mathrm{O}_{2} \leq 15 \%$ (resultados não apresentados) não se observou significativa redução da intensidade do íon referente a AME, na maioria dos experimentos a intensidade dos demais íons formados alcançou menos de $5 \%$ da intensidade do íon principal $(\mathrm{m} / \mathrm{z}$ 228) e perto da faixa de ruído no espectro de massas, não sendo possível utilizá-los para compor os resultados. Nos experimentos com quantidade de $\mathrm{H}_{2} \mathrm{O}_{2} \geq$ $25 \%$ e nas temperaturas e $\mathrm{pH}$ estudados apresentaram uma intensa oxidação da AME e dos seus subprodutos de oxidação (resultados não apresentados), não sendo possível determinar os íons formados devido a numerosa formação de íons com baixa intensidade. Diante do exposto, as melhores condições para identificação dos produtos de oxidação da $\mathrm{AME}$ foram determinados na temperatura de $65^{\circ} \mathrm{C}$ no pH 1,3 e 7 com $20 \%$ e $25 \%$ de $\mathrm{H}_{2} \mathrm{O}_{2}$ em experimentos de 10 horas de duração.

Os primeiros ensaios utilizando a Reação de Fenton foram realizados em $\mathrm{pH}$ 1 com $20 \%$ e $25 \%$ de $\mathrm{H}_{2} \mathrm{O}_{2}$ na temperatura de $65{ }^{\circ} \mathrm{C}$, sendo registrados os valores de 
intensidades dos íons $\mathrm{s}^{-1}$ mais abundantes,

conforme mostra a Tabela 1 .

Tabela 1 - Intensidade dos íons $\left(\mathrm{s}^{-1}\right)$ da molécula da Ametrina e de seus subprodutos de degradação em pH 1, temperatura de $65^{\circ} \mathrm{C}$ e concentrações de $25 \%$ de $\mathrm{H}_{2} \mathrm{O}_{2}$

\begin{tabular}{|c|c|c|}
\hline \multicolumn{3}{|c|}{ Intensidade dos íons (íons s ${ }^{-1}$ ) } \\
\hline \multirow{2}{*}{$\begin{array}{c}\text { tempo } \\
\text { min. }\end{array}$} & \multicolumn{2}{|c|}{$\mathrm{H}_{2} \mathrm{O}_{2} 25 \%$} \\
\cline { 2 - 3 } & Ametrina & $\mathrm{m} / \mathrm{z} 185$ \\
\hline 0 & $7,0 \times 10^{6}$ & $\mathrm{ND}$ \\
\hline 15 & $3,5 \times 10^{6}$ & $\mathrm{ND}$ \\
\hline 30 & $2,4 \times 10^{6}$ & $6,0 \times 10^{5}$ \\
\hline 45 & $2,0 \times 10^{6}$ & $9,0 \times 10^{5}$ \\
\hline 60 & $1,7 \times 10^{6}$ & $6,6 \times 10^{5}$ \\
\hline 90 & $1,0 \times 10^{6}$ & $4,0 \times 10^{5}$ \\
\hline 120 & $9,5 \times 10^{5}$ & $1,3 \times 10^{5}$ \\
\hline 600 & $1,6 \times 10^{5}$ & $\mathrm{ND}$ \\
\hline \multicolumn{3}{|c|}{$* \mathrm{ND}=$ não detectado } \\
\hline
\end{tabular}

De acordo com a Tabela 1, pode-se observar a redução na intensidade do sinal correspondente à molécula de AME. A partir de 30 minutos de reação, ocorre a formação do subproduto $\mathrm{m} / \mathrm{z} 185$, com o aumento da sua intensidade até $45 \mathrm{~min}$ com posterior diminuição da sua intensidade até o final do experimento, indicando que sua estrutura pode estar sendo oxidada. Este subproduto é resultado de reações de adição nucleofílica do $\bullet \mathrm{OH}$ ao $\mathrm{C}_{\alpha}$ de um dos grupamentos alquílicos pertencentes às aminas, seguido pela eliminação do grupamento amina, conforme Figura 2.

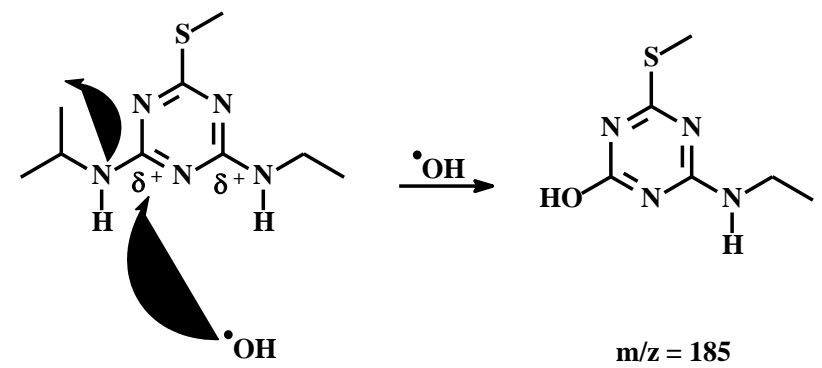

Figura 2. Subprodutos da oxidação da AME em pH 1, temperatura de $65^{\circ} \mathrm{C}$ e $25 \%$ de $\mathrm{H}_{2} \mathrm{O}_{2}$.

Com os dados obtidos com o processo Fenton em $\mathrm{pH} 1$ pode-se observar a oxidação da molécula da AME formando um subproduto de massa $\mathrm{m} / \mathrm{z}, 185$, porém, como descrito na literatura, a melhor faixa de $\mathrm{pH}$ para o processo Fenton é próxima a $\mathrm{pH}$ 3, assim, para os próximos experimentos de oxidação da AME a solução foi ajustada em pH 3 (NOGUEIRA 2004). A exemplo dos experimentos anteriores em $\mathrm{pH} 1$, os experimentos para oxidação da AME em pH 3 apresentou mínima formação de subprodutos nos experimentos com quantidade de $\mathrm{H}_{2} \mathrm{O}_{2} \leq$ $15 \%$ e com $\mathrm{H}_{2} \mathrm{O}_{2} \geq 25 \%$ ocorreu massiva oxidação da AME com extensa formação de produtos com baixa intensidade, assim, os experimentos em pH 3 com $20 \%$ de $\mathrm{H}_{2} \mathrm{O}_{2}$ apresentaram a maior quantidade de produtos formados em intensidade passiveis de consideração para análise da 
oxidação da AME, conforme pode ser observado na Tabela 2.

Tabela 2 - Intensidade dos íons $\mathrm{s}^{-1}$ da molécula da Ametrina e de seus subprodutos de degradação em $\mathrm{pH} 3$, temperatura de $65{ }^{\circ} \mathrm{C}$ e concentrações de $20 \%$ de $\mathrm{H}_{2} \mathrm{O}_{2}$

\begin{tabular}{|c|c|c|c|}
\hline \multicolumn{4}{|c|}{ Intensidade dos íons (íons s ${ }^{-1}$ ) } \\
\hline \multirow{2}{*}{$\begin{array}{c}\text { tempo } \\
\text { min }\end{array}$} & \multicolumn{3}{|c|}{$\mathrm{H}_{2} \mathrm{O}_{2} 20 \%$} \\
\cline { 2 - 4 } & Ametrina & $\mathrm{m} / \mathrm{z} 185$ & $\mathrm{~m} / \mathrm{z} 197$ \\
\hline 0 & $7,0 \times 10^{6}$ & $\mathrm{ND}$ & $\mathrm{ND}$ \\
\hline 15 & $4,0 \times 10^{6}$ & $7,0 \times 10^{5}$ & $\mathrm{ND}$ \\
\hline 30 & $2,8 \times 10^{6}$ & $9,0 \times 10^{5}$ & $7,0 \times 10^{6}$ \\
\hline 45 & $9,5 \times 10^{5}$ & $5,0 \times 10^{5}$ & $4,0 \times 10^{6}$ \\
\hline 60 & $9,2 \times 10^{5}$ & $3,0 \times 10^{5}$ & $1,0 \times 10^{6}$ \\
\hline 90 & $8,9 \times 10^{5}$ & $7,0 \times 10^{5}$ & $5,0 \times 10^{5}$ \\
\hline 120 & $6,3 \times 10^{5}$ & $9,0 \times 10^{5}$ & $4,5 \times 10^{5}$ \\
\hline 600 & $2,8 \times 10^{5}$ & $\mathrm{ND}$ & $\mathrm{ND}$ \\
\hline \multicolumn{4}{|c|}{$* \mathrm{ND}=$ não detectado } \\
\hline
\end{tabular}

Segundo a Tabela 2 pode se observar formação de dois possíveis subprodutos de oxidação, $\mathrm{m} / \mathrm{z}=197 \mathrm{e} \mathrm{m} / \mathrm{z}$ $=185$. O fragmento $\mathrm{m} / \mathrm{z} 185$, como descrito anteriormente, é formado pela adição nucleofílica do ${ }^{\circ} \mathrm{OH}$ ao $\mathrm{C}_{\alpha}$ com eliminação do grupamento amina, segundo a Figura 2. Enquanto o fragmento $\mathrm{m} / \mathrm{z}=$
197 corresponde ao subproduto de oxidação da AME formado pela dessulfurização, remoção do grupamento $\mathrm{SCH}_{3}$, seguido por uma hidroxilação, conforme a Figura 3.<smiles>CCN(C)c1nc(NC)nc(N(C)C(C)C)n1</smiles><smiles>CCNc1nc(O)nc(N([Tl])C(C)C)n1</smiles>

produto de degradação

Figura 3. Subproduto da oxidação da $\mathrm{AME}$ em pH 3, temperatura de $65{ }^{\circ} \mathrm{C}$ e $20 \%$ de $\mathrm{H}_{2} \mathrm{O}_{2}$.

De acordo com a literatura (BRIX 2009; SEFFERNICK 2000), o radical hidroxila é formado em reações que combinam o peróxido de hidrogênio e catalisadores, e conforme o contaminante, diferentes interações envolvendo o radical hidroxila, tais como saída de átomos de hidrogênio, reações nucleofílicas ou até mesmo a transferência eletrônica e reações radical-radical (BRILLAS 2009).

Para maiores informações sobre o processo de oxidação da AME, foram realizados experimentos de oxidação em condições mais brandas, isto é, em pH 7 e temperatura de $65{ }^{\circ} \mathrm{C}$. Os resultados para este tipo de ensaio foram favoráveis se considerarmos que todos os fragmentos vistos anteriormente foram detectados, e ainda foi possível identificar novos fragmentos da oxidação da AME. Para as amostras do ensaio em $\mathrm{pH} 7$ pudessem ser analisadas adequadamente pelo módulo ESI, esperou-se precipitar o $\mathrm{Fe}^{3+}$, sendo que o precipitado não foi analisado devido 
à alta quantidade de metal, o que não descarta a possibilidade de neste precipitado conter matéria orgânica. As Tabelas 3A e 3B mostram a variação de intensidade da AME e de seus subprodutos de oxidação nos experimentos com $20 \%$ e $25 \%$ de $\mathrm{H}_{2} \mathrm{O}_{2}$.

Tabela 3A - Intensidade dos íons $\mathrm{s}^{-1}$ da molécula da Ametrina e de seus subprodutos de degradação em pH 7, temperatura de $65^{\circ} \mathrm{C}$ e $20 \%$ de $\mathrm{H}_{2} \mathrm{O}_{2}$

\begin{tabular}{|c|c|c|c|}
\hline \multicolumn{4}{|c|}{ Intensidade dos íons (íons $\mathrm{s}^{-1}$ ) } \\
\hline \multirow{2}{*}{$\begin{array}{c}\text { tempo } \\
\text { min }\end{array}$} & Ametrina & $\mathrm{m} / \mathrm{z} 197$ & $\mathrm{~m} / \mathrm{z} 199$ \\
\hline 0 & $7,0 \times 10^{6}$ & $\mathrm{ND}$ & $\mathrm{ND}$ \\
\hline 15 & $6,3 \times 10^{6}$ & $1,6 \times 10^{6}$ & $3,0 \times 10^{6}$ \\
\hline 30 & $5,6 \times 10^{6}$ & $1,3 \times 10^{6}$ & $5,0 \times 10^{6}$ \\
\hline 45 & $5,3 \times 10^{6}$ & $1,5 \times 10^{6}$ & $2,3 \times 10^{6}$ \\
\hline 60 & $4,7 \times 10^{6}$ & $1,0 \times 10^{6}$ & $1,5 \times 10^{6}$ \\
\hline 90 & $2,2 \times 10^{6}$ & $8,5 \times 10^{5}$ & $7,5 \times 10^{5}$ \\
\hline 120 & $2,0 \times 10^{6}$ & $5,0 \times 10^{5}$ & $4,5 \times 10^{5}$ \\
\hline 600 & \multicolumn{4}{|c}{$1,0 \times 10^{6}$} & $1,0 \times 10^{5}$ & $1,0 \times 10^{5}$ \\
\hline \multicolumn{4}{|c}{ * ND = não detectado } \\
\hline
\end{tabular}

A partir dos espectros de massas da AME em pH 7 e $20 \%$ de $\mathrm{H}_{2} \mathrm{O}_{2}$ pode-se identificar os subprodutos $\mathrm{m} / \mathrm{z}=197$ e $\mathrm{m} / \mathrm{z}$ 199 (Tabela 3A). O fragmento $\mathrm{m} / \mathrm{z}=197$ corresponde à formação do produto de oxidação resultante da desssulfurização, seguida de hidroxilação, conforme Figura 3. Enquanto o fragmento $\mathrm{m} / \mathrm{z}=199$ corresponde a hidroxilação de uma das extremidades amina da AME, seguida pela eliminação de $-\mathrm{NCH}_{2} \mathrm{CH}_{3}$, conforme Figura 4.

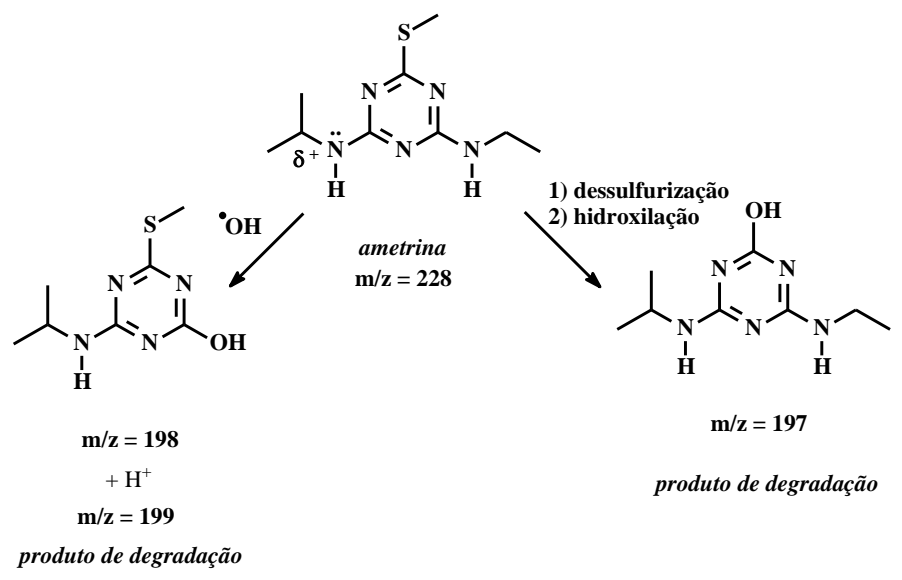

Figura 4. Subproduto da oxidação da $\mathrm{AME}$ em pH 7, temperatura de $65^{\circ} \mathrm{C}$ e $20 \%$ de $\mathrm{H}_{2} \mathrm{O}_{2}$.

O aumento da proporção de $20 \%$ para $25 \%$ de $\mathrm{H}_{2} \mathrm{O}_{2}$ proporcionou $\mathrm{O}$ surgimento de mais um produto de oxidação da AME, confirmado pelo fragmento $\mathrm{m} / \mathrm{z}=243$, conforme mostra a Tabela 3B.

Tabela 3B - Intensidade dos íons $\mathrm{s}^{-1}$ da molécula da Ametrina e de seus subprodutos de degradação em pH 7, temperatura de $65{ }^{\circ} \mathrm{C}$ e $25 \%$ de $\mathrm{H}_{2} \mathrm{O}_{2}$ 


\begin{tabular}{|c|c|c|c|c|}
\hline \multicolumn{5}{|c|}{ Intensidade dos íons (íons s ${ }^{-1}$ ) } \\
\hline \multirow[b]{2}{*}{$\begin{array}{c}\text { tempo } \\
\min \end{array}$} & \multicolumn{4}{|c|}{$\mathrm{H}_{2} \mathrm{O}_{2} 25 \%$} \\
\hline & Ametrina & $\begin{array}{l}\mathrm{m} / \mathrm{z} \\
185\end{array}$ & $\begin{array}{c}\mathrm{m} / \mathrm{z} \\
199\end{array}$ & $\begin{array}{l}\mathrm{m} / \mathrm{z} \\
243\end{array}$ \\
\hline 0 & $7,0 \times 10^{6}$ & ND & ND & ND \\
\hline 15 & $5,8 \times 10^{6}$ & $6,0 \times 10^{5}$ & $1,4 \times 10^{6}$ & $3,0 \times 10^{5}$ \\
\hline 30 & $5,4 \times 10^{6}$ & $9,0 \times 10^{5}$ & $1,3 \times 10^{6}$ & $3,0 \times 10^{5}$ \\
\hline 45 & $4,9 \times 10^{6}$ & $9,0 \times 10^{5}$ & $1,0 \times 10^{6}$ & $3,5 \times 10^{5}$ \\
\hline 60 & $3,3 \times 10^{6}$ & $4,0 \times 10^{5}$ & $8,2 \times 10^{5}$ & $1,0 \times 10^{6}$ \\
\hline 90 & $1,8 \times 10^{6}$ & ND & $3,5 \times 10^{5}$ & $2,0 \times 10^{5}$ \\
\hline 120 & $1,1 \times 10^{6}$ & ND & ND & ND \\
\hline 600 & $8,6 \times 10^{5}$ & ND & ND & ND \\
\hline
\end{tabular}

Observa-se na molécula da AME que o carbono localizado na posição $\alpha$ ao grupamento amina e ligado a uma isopropila, apresenta uma densidade de carga positiva $\left(\delta^{+}\right)$, devido sua<smiles>CCNc1nc(SC)nc(N([Tl])C(C)C)n1</smiles>

proximidade ao átomo de nitrogênio. Dessa forma, este $C_{\alpha}$ pode sofrer ataque nucleofílico do radical hidroxila, gerando o subproduto $\mathrm{m} / \mathrm{z}=243$ e liberando um hidreto.

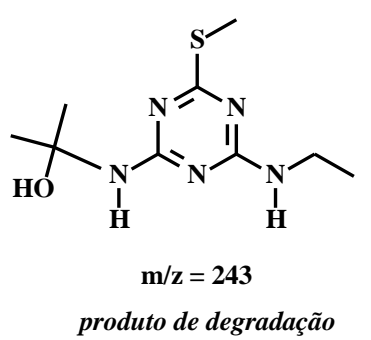

Figura 5. Subproduto da oxidação da AME em pH 7, temperatura de $65{ }^{\circ} \mathrm{C}$ e $25 \%$ de $\mathrm{H}_{2} \mathrm{O}_{2}$.

Observando os espectros da oxidação da AME, nota-se que o produto de $m / z, 243$ apresenta a relação $m / z$ superior à da $\mathrm{AME}$, o que pode ser explicado pela a adição eletrofílica de radical hidroxila a compostos orgânicos que contêm ligações $\pi$ ou pares eletrônicos que possam ser deslocalizados (alta eletronegatividade) resulta na formação de radicais orgânicos, com massas superiores a inicial (NEYENS 2003; WALLING 1975).

\section{Proposta de uma Rota de Degradação da AME}

Segundo o mecanismo de oxidação da AME de uma maneira geral, os subprodutos formados estão relacionados diretamente com as características estruturais do composto, ou seja, seus grupos funcionais reativos. Considerando os produtos obtidos a partir da oxidação da
ATZ foi possível prever que as reações de fragmentação da AME ocorre inicialmente a partir da retirada de grupos alquil (reação de desalquilação) iniciada pelo o radical peróxido, seguida da reação hidroxilação, logo esta reação pode ser de substituição nucleofílica, mas não é possível prever se esta ocorre por $\mathrm{SN}^{1}$ ou $\mathrm{SN}^{2}$, pois como já se conhece as reações por peróxido catalisado não são seletivas.

Diante do processo de oxidação da AME e formação de diferentes subprodutos, nota-se que a intensidade do íon referente a AME apresentou diminuição em todos os experimentos, porém, dependo do $\mathrm{pH}$ e da temperatura utilizada em cada experimento foi alcançada uma remoção da AME, conforme pode ser observada na Figura 6. 


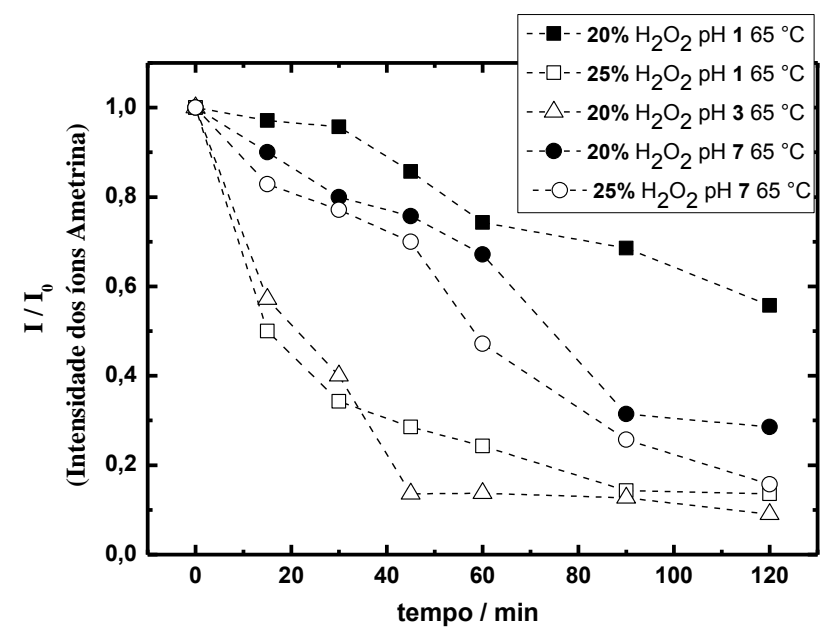

Figura 6. Decaimento da intensidade de detecção da Ametrina nos experimentos em diferentes $\mathrm{pH}$, com $20 \%$ e $25 \%$ de $\mathrm{H}_{2} \mathrm{O}_{2}$ e temperatura de $65^{\circ} \mathrm{C}$.

A Figura 6 mostra o decaimento normalizado da intensidade de detecção da AME em todos os processos estudados, onde, comparando o decaimento da molécula da AME com os processos de degradação realizados, podemos verificar que o processo que permitiu a maior detecção de subprodutos foi na condição experimental com $\mathrm{pH} 7$ e nas proporções de degradação de $20 \%$ e $25 \%$ de $\mathrm{H}_{2} \mathrm{O}_{2}$. Nota-se que os experimentos com pH 7 e $\mathrm{pH} 1\left(20 \%\right.$ de $\left.\mathrm{H}_{2} \mathrm{O}_{2}\right)$ apresentaram a menores reduções nas intensidades do íon $\mathrm{m} / \mathrm{z} 228$ referente a AME. Nos experimentos em $\mathrm{pH} 1\left(25 \%\right.$ de $\left.\mathrm{H}_{2} \mathrm{O}_{2}\right)$ e $\mathrm{pH}$ 3 apresentaram maior remoção da MAE, alcançando as maiores remoção em menos de $100 \mathrm{~min}$ de experimento, mostrando a efetividade do meio ácido para a oxidação da AME.

Com os resultados apresentados foram previstos os produtos de oxidação da ametrina utilizando Reação de Fenton com $20 \%$ e $25 \%$ de $\mathrm{H}_{2} \mathrm{O}_{2}$ em pH 1, 3 e 7 na temperatura $65{ }^{\circ} \mathrm{C}$, conforme podo ser observado na Figura 7. 
<smiles>Oc1nc(O)nc(O)n1</smiles>

ND<smiles>CSc1nc(O)nc(O)n1</smiles>

$\mathbf{m} / \mathbf{z}=159$

ND<smiles>CCNc1nc(O)nc(NC(C)C)n1</smiles>

$\mathbf{m} / \mathbf{z}=197$

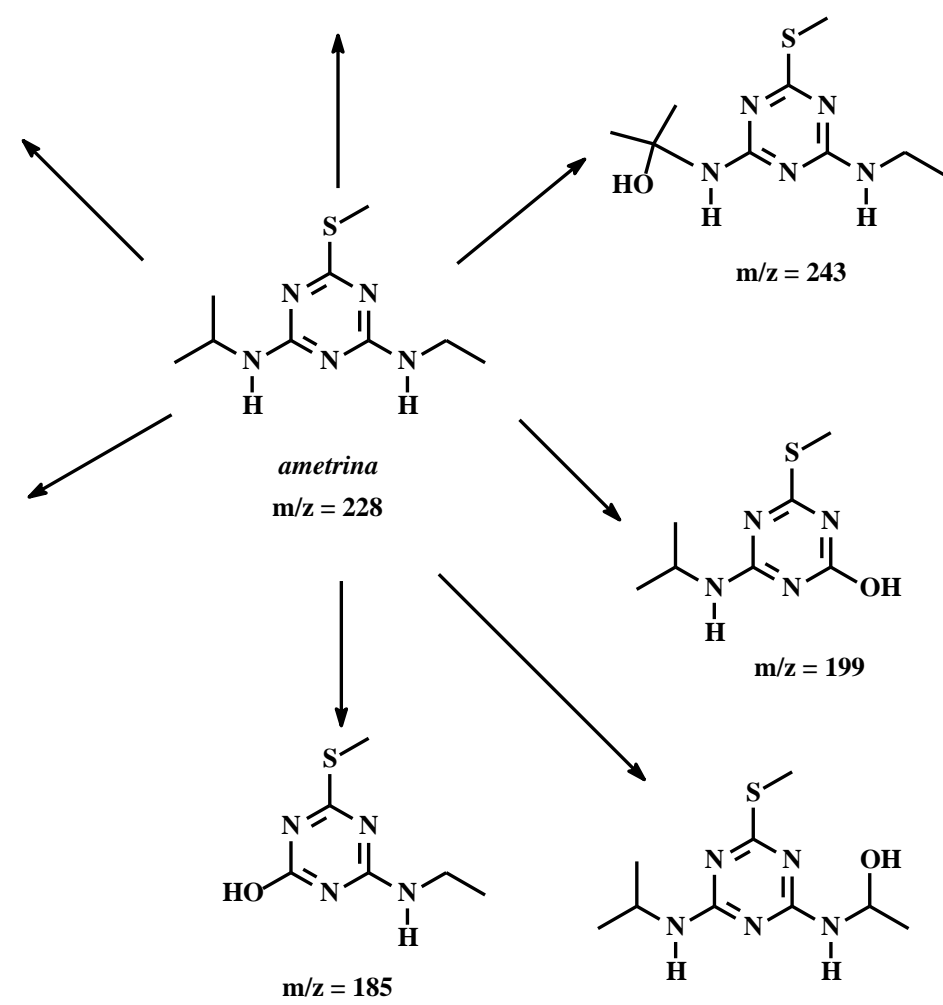

$\mathrm{m} / \mathrm{z}=\mathbf{2 4 3}$

Figura 7. Previsão dos possíveis produtos da oxidação da AME.

Com os resultados obtidos com o processo de oxidação controlada da AME, a Figura 7 apresenta uma previsão dos produtos formados durante os diferentes experimentos de oxidação estudados. Notase que todos os subprodutos previstos são originados na AME, mesmo em experimentos com 10 horas de duração, o

\section{CONCLUSÕES}

O estudo da oxidação do herbicida ametrina foi realizado em diferentes tipos de experimentos. A variação de $\mathrm{pH}$ e temperatura não proporcionou formação de novos subprodutos a partir da oxidação da ametrina, esse comportamento é que denota a estabilidade desse composto no meio ambiente, indicando que para se alcançar uma maior oxidação são necessárias condições experimentais mais agressivas, e assim se obter real redução da carga orgânica em sistemas contaminados com ametrina.

condizente com a proposta desse tipo de composto, ser estável no meio ambiente.

Diante dessa estabilidade foram realizados experimentos utilizando $\mathrm{POA}$, com diferentes quantidades de $\mathrm{H}_{2} \mathrm{O}_{2}$ e diferentes temperaturas e $\mathrm{pH}$. Os resultados obtidos mostraram que 
concentrações menores a $15 \%$ de $\mathrm{H}_{2} \mathrm{O}_{2}$ não foram suficientes para oxidar a AME, enquanto que os experimentos em que foram utilizadas concentrações superiores a $30 \%$ promoveram massiva oxidação do composto, não sendo possível determinar os subprodutos formados.

Nesse sentido, os experimentos com $20 \%$ e $25 \%$ de $\mathrm{H}_{2} \mathrm{O}_{2}$ em $65{ }^{\circ} \mathrm{C}$ apresentaram as melhores respostas para identificação dos subprodutos de oxidação da ametrina, com possíveis 5 subprodutos determinados, porém todos originados diretamente da molécula da AME. Esses possíveis subprodutos determinados mostram que mesmo em condições

\section{AGRADECIMENTOS}

Os autores agradecem à Fundação de Amparo à Pesquisa do Estado de São Paulo (FAPESP) (processos $\mathrm{n}^{\circ}$ 2013/02762-5; 2007/03774-6; 2014/50945$4 ; 2016 / 01937-4)$ e ao Conselho Nacional

\section{REFERÊNCIAS}

BRILLAS, E.; SIRÉS, I.; OTURAN, M. A.; Electro-Fenton Process and Related Electrochemical Technologies Based on Fenton's Reaction Chemistry. Chem. Rev. v. 109(12): 6570-6631, 2009.

BRIX, R.; BAHI, N.; ALDA, M. J. L.; FARRÉ, M.; FERNANDEZ, J-M.; BARCELÓ, D.; Identification of disinfection by-products of selected triazines in drinking water by LC-Q-ToF-MS/MS and evaluation of their toxicity. J. Mass. Spectrom, v. 44: 330337, 2009.

Conselho Nacional do Meio Ambiente. Resolução no. 357 de 17 de março de 2005.

Ministério do meio Ambiante, 2005.

DICTOR, M-C.; BARAN, N.; GAUTIER, A.; MOUVET, C.; Acetochlor mineralization and fate of its two major metabolites in two soils under laboratory conditions. Chemosphere, v. 71(4): 663-670, 2008.

EL IMACHE, A.; DAHCHOUR, A.; ELAMRANI, B.; DOUSSET, S.; oxidativas, a molécula da AME possui relativa estabilidade, pois foram necessárias 10 horas de experimento, na melhor condição $\left(20 \%\right.$ de $\mathrm{H}_{2} \mathrm{O}_{2}$ e pH 3$)$, para se alcançar aproximadamente $90 \%$ de remoção da AME.

Os resultados empregados neste trabalho podem ser utilizados para ajudar a alertar sobre o potencial de mobilidade dos agrotóxicos e da possibilidade de contaminação das águas subterrâneas, fornecendo subsídios para o desenvolvimento de processos de tratamento via POA de águas contaminadas com ametrina.

de Desenvolvimento Científico e Tecnológico (CNPq) (processos $\mathrm{n}^{\mathrm{o}}$ 446436/2014-3; 301492/2013-1; 465571/2014-0 (INCT-DATREM)) pelo auxílio financeiro.

POZZONNI, F.; GUZZELLA, L.; Leaching of Diuron, Linuron and their main metabolites in undisturbed field lysimeters. J. Environ. Sci. Health, Part B, v. 44: 31-37, 2009.

FARRÉ, M.; FERNANDEZ, J.; PAEZ, M.; GRANADA, L.; BARBA, L.; GUTIERREZ, H. M.; PULGARIN, C.; Barceló, D. Analysis and toxicity of methomyl and ametryn after biodegradation. Anal. Bioanal. Chem, v. 373(8): 704-709, 2002.

FAVA, F.; ORRÙ, M. A.; SCARDALA, S.; FUNARI, E.; Leaching potential of carbamates and their metabolites and comparison with triazines. Microchem J. v. 86: 204-208, 2007.

GAO, N-Y.; DENG, Y.; ZHAO, D.; Ametryn degradation in the ultraviolet (UV) irradiation/hydrogen peroxide (H2O2) treatment. J. Hazard. Mat. v. 164: 640-645, 2009. 
GRILLO, R.; SANTOS, N. Z. P.; MARUYAMA, C. R.; ROSA, A. H.; LIMA, R.; FRACETO, L. F.; Poly $(\varepsilon-$ caprolactone)nanocapsules as carrier systems for herbicides: Physico-chemical characterization and genotoxicity evaluation J. Hazard. Mat. v. 231-232: 19, 2012.

JABLONOWSKI, N. D.; KOEPPCHEN, S.; HOFMANN, D.; SSHAEFFER, A.; BURAEL, P.; Spatial Distribution and Characterization of Long-Term Aged ${ }^{14} \mathrm{C}$ Labeled Atrazine Residues in Soil. J. Agric. Food Chem, v. 56: 9548-9554, 2008.

LENNART, T.; HARALD, C.; ELIZABET, B.; JOHN, S.; Environmental problems with the use of diuron on Swedish railways. Pestic. Outlook. v. 13(3): 108-115, 2002.

MASCOLO, G.; LOPES, A.; PASSINO, R.; RICCO, G.; TIRAVANTI, G.; Degradation of sulphur containing striazines during water chlorination, Water Res. v. 28(12): 2499-2506, 1994.

\section{MELGOSA, D.; HERNANDEZ- RAMIREZ, A.; PERALTA- HERNANDEZ, J. M.; Comparative efficiencies of the decolourisation of Methylene Blue using Fenton's and photo-Fenton's reactions. Photochem. Photobiol. Sci. v. 8(5): 596-599, 2009.}

NAVARATNA, $\quad$ D.; $\quad$ SHU, L.; BASKARAN, K.; JEGATHEESAN, V.; Model development and parameter estimation for a hybrid submerged membrane bioreactor treating Ametryn. Bioresource Technology, 113: 191-200, 2012.

NEYENS, E.; BAYEANS, J.; A review of classic Fenton's peroxidation as an advanced oxidation technique J. Hazard. Mat. v. B98: 33-50, 2003.

NOGUEIRA, R. F. P.; TROVÓ, A. G.; SILVA, M. R. A.; OLIVEIRA, M. C.; VILLA, R. D.; Fundaments and Environmental applications of fenton and phto-fenton processes. Quim. Nova. V. 30(2): 400-408, 2007.

NOGUEIRO, R. F. P.; TROVÓ, A. G.; MODÉ, D. F.; Solar photodegradation of dichloroacetic acid and 2,4-dichlorophenol using an enhanced photo-Fenton process. Chemosphere, v. 48(4): 385-391, 2002.

NOGUERIA, R. F. P.; TROVÓ, A. G.; PATERLINI, W. C.; Evaluation of the combined solar $\mathrm{TiO}_{2}$ /photo-Fenton process using multivariate analysis. Water Sci. Technol. v. 49(4): 195-200, 2004.

OTURAN, M. A.; An ecologically effective water treatment technique using electrochemically generated hydroxyl radicals for in situ destruction of organic pollutants: Application to herbicide 2,4-D. J. Appl. Electrochem. v. 30(4): 475-482, 2000

OTURAN, M. A.; OTURAN, N.; EDELAHI, M. C.; PODVARICA, F. I.; El KACEMI, K.; Oxidative degradation of herbicide diuron in aqueous medium by Fenton's reaction based advanced oxidation processes. Chem. Eng. J. v. 171(1): 127-135, 2011.

REBELO, S. L. H.; MELO, A.; COIMBRA, R.; AZENHA, M. E.; PEREOIRA, M. M.; BURROWS, H. D.; SARAKHA, M.; Photodegradation of atrazine and ametryn with visible light using water soluble porphyrins as sensitizers. Environ. Chem. Lett. V. 5(1): 29-33, 2007.

REZENDE, E. I. P.; PERALTAZANORA, P. G.; ABATE, G.; Sorption Study of herbicides with the cla minerals vermiculite and montmorillonite. Quím. Nova, v. 34(1): 21-27, 2001.

SEFFERNICK, J. L.; JOHNSON, G.; Sadoesky, M. J.; WACKETT, L. P.; Substrate Specificity of Atrazine Chlorohydrolase and AtrazineCatabolizing Bacteria. Appl. Env. Microbiology, v. 66(10): 4247-4252, 2000.

SPADOTO, C. A.; GOMES, M. A. F. Agrotóxicos no Brasil. Agência 
EMBRAPA de Informação Tecnológica, 2015. Disponível em: $<$ http://www.agencia.cnptia.embrapa.br/ge stor/agricultura_e_meio_ambiente/arvore/ CONTAG01_40_210200792814.html>.

TROVÓ, A. G.; VILLA, R. D.; NOGUEIRA, R. F. P.; Use of photo-fenton reactions in the prevention of agricultural contaminations. Quim. Nova v. 28(5): 847-851, 2005.

WALLING, C.; Fenton's Reagent Revisited. Acc. Chem. Res. v. 8(4): 125$131,1975$.

WALLING, C.; GOOSEN, A.; Mechanism of the Ferric Ion Catalyzed Decomposition of Hydrogen Peroxide. Effect of Organic Substrates. J. Am. Chem. Soc. v. 95(11): 2997-2991, 1973.

XU, B.; GAO, N.; CHENG, H.; HU, C.; XIA, S.; SUN, X.; WANG, X.; YANG, S.; Ametryn degradation by aqueous chlorine: Kinetics and reaction influences $\mathbf{J}$. Hazard. Mat. 169: 586-592, 2009.

XU, B.; GAO, N-y.; CHENG, H.; HU, CY.; XUA, S-J.; SUN, X-F.; WAG, X.; YANG, S. Ametryn degradation by aqueous chlorine: Kinetics and reaction influences. J. Hazard. Mat, v. 169: 586592, 2009.

ZALOUK, S.; BASBATI, S.; SERGENT, M.; AMBROSIO, M.; Disposal of animal by-products by wet air oxidation: Performance optimization and kinetics. Chemosphere. v. 74(2): 193-199, 2009. 